\title{
Assessment of Isocitrate Dehydrogenase 1 Mutation by Immunohistochemistry in Egyptian Patients with High-grade Gliomas
}

\author{
Essam Ayad ${ }^{1}$, Sylvia Mikhael Ghattas ${ }^{1}$, Rabab Abdel Moneim², Azzam Ismail ${ }^{3}$, Rasha A. Khairy ${ }^{1 *}$ \\ ${ }^{1}$ Department of Pathology, Faculty of Medicine, Cairo University, Giza, Egypt; ${ }^{2}$ Clinical Oncology, Faculty of Medicine, Cairo \\ University, Giza, Egypt; ${ }^{3}$ Department of Histopathology, Leeds Teaching Hospital, Leeds, England
}

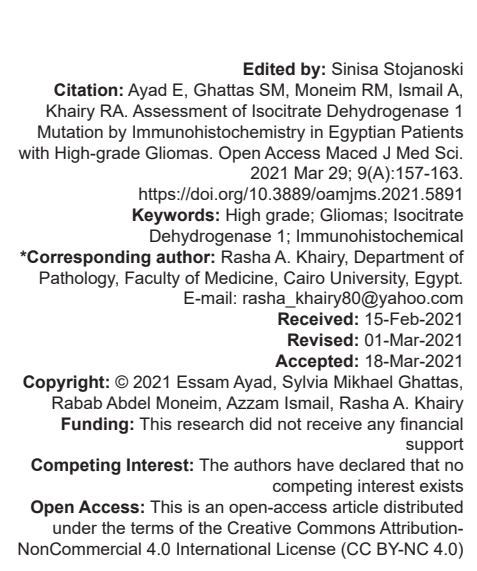

Introduction

Molecular factors were recently introduced in the classification of glioma cases in the latest 2016 classification of central nervous system (CNS) tumors by the World Health Organization (WHO) [1].

Diffuse astrocytomas (WHO Grade II) and anaplastic astrocytomas (WHO Grade III) are reclassified into isocitrate dehydrogenase (IDH) mutant, IDH wild type, and nitric oxide synthases (NOS) variants. Most Grade II and III gliomas are reported to be IDH mutant if IDH testing is applied. IDH wild type is reported if immunohistochemistry (IHC) for mutant $\mathrm{R} 132 \mathrm{H}$ IDH1 protein and sequencing for IDH1 codon 132 and IDH2 codon 172 gene mutations yield negative results or if sequencing for IDH1 codon 132 and IDH2 codon 172 gene mutations alone is negative [2].
Glioblastoma (WHO Grade IV) in the latest 2016 WHO CNS classification is reclassified into (1) glioblastoma, IDH wild type, which accounts for nearly $90 \%$ of the cases and is usually primary or de novo glioblastomas, (2) glioblastoma, IDH mutant, which accounts for nearly $10 \%$ of cases and is usually secondary glioblastomas arising on top of lower grade diffuse gliomas and usually affects younger age group, and (3) glioblastoma, NOS, is termed when complete IDH evaluation is not applicable [1].

IDH mutations are clustered at codon 132 of IDH1 and codon 172 of the IDH2 gene, suggesting that they acquire a gain of function to the mutant enzymes. These mutations eventually lead to mutated IDH protein production which will subsequently result in production of higher levels of D-2-hydroxyglutarate, instead of a-ketoglutarate. D-2-hydroxyglutarate is an oncometabolite which results in epigenetic 
aberrations that are incorporated in the early steps of gliomagenesis [3], [4], [5].

On clinical settings, the existence of IDH1 mutation is associated with better outcome in glioma patients of all grades [6], [7]. Interestingly, also in conjunction with 0-6-Methylguanine-DNA methyltransferase (MGMT) promoter methylation status, IDH1 mutation seems to play a role in the prognosis and in the prediction of response to chemotherapy [8]. Subsequently, IDH1 mutation may have a direct impact on treatment decisions. Therefore, accurate evaluation of IDH1 mutational status is increasingly promoted. To date, the "gold standard" for the assessment of IDH1 mutation is DNA sequencing, yet with limited sensitivity results and not easy applied in most laboratories. Therefore, immunohistochemical analysis of IDH1 was currently introduced as a practical alternative method with high sensitivity and specificity results. Moreover, IHC allows for quantitative analysis of IDH1 mutation which was not feasible before [9], [10].

We aimed in this work to apply IHC as an analytical method for the assessment of IDH1 mutation and to correlate the results of IDH1 mutation by immunohistochemical analysis with different clinicopathologic parameters and disease course.

\section{Patients and Methods}

This retrospective study was preformed following protocol approval by the collaboration of the ethical committee at the Pathology and Oncology Departments in Faculty of Medicine, Cairo University.

Inclusion criteria were cases of high-grade gliomas (Grades III and IV) based on histopathologic diagnosis, availability of the relevant clinical data, and availability of the follow-up data.

Exclusion criteria were paucity of tumor tissue in paraffin blocks, defective clinical or follow-up data.

The material of this work consisted of 60 cases diagnosed as high-grade glioma including anaplastic astrocytoma (5 cases), anaplastic oligodendroglioma ( 3 cases), and glioblastoma (52 cases). The paraffin blocks were retrieved from the Pathology Department, Faculty of Medicine, Cairo University, for patients diagnosed over 2-year period from January 2015 to January 2017, to fulfill a minimum follow-up period of 2 years duration. The personal data, clinical details, and pathological data pertaining to these patients were retrieved from the medical records at Faculty of Medicine, Cairo University.

The demographic and clinicopathologic data reported from the medical records were age and gender of the patients, site and size of brain tumor, presence of neurologic sensory and motor symptoms, and status of intracranial tension. The performance status (PS) was scored according to Zubrod scale [11]; PS scores 0, 1, and 2 were reported as good PS, while scores 3 and 4 were reported as poor $\mathrm{PS}$.

\section{evaluation \\ Response to treatment criteria and}

All of the patients (60 patients) underwent tissue sampling (with surgical resection whenever possible) and were followed up for 2 years or more.

Fifty-seven patients received radiotherapy treatment. The response to radiotherapy treatment was recorded as stationary, progression, partial remission, and complete remission according to the results of posttreatment magnetic resonance imaging (MRI) scan.

Forty-seven patients received adjuvant chemotherapy treatment. The response to chemotherapy treatment was recorded as stationary, progression, partial remission, and complete remission according to the results of post-treatment MRI scan.

\section{Survival analysis}

Overall survival (OS) and progression-free survival (PFS) were calculated for all patients included in the current study (60 patients). All data for OS and PFS were analyzed by January 31, 2019.

OS was calculated from the date of histological diagnosis until the date of death from any cause [12].

PFS was defined as the time from histological diagnosis until the date of disease progression (radiologic evidence of disease progression on $\mathrm{MRI}$ ) or the date of death from any cause or last follow-up whichever was earlier [12].

\section{Histopathologic and immunohistochemical}

\section{staining}

The paraffin blocks of the 60 cases included in the study were retrieved from the pathology departmental archives. Two sections were cut from each paraffin block by microtome at 5 microns thickness; one stained with hematoxylin and eosin for routine histopathological examination and confirmation of diagnosis according to latest WHO classification criteria [1]. The other one was mounted on positively charged slides for immunohistochemical analysis of proliferating astrocytic cells and oligodendroglial cells by mouse monoclonal antibody $(\mathrm{H} 09)$ to IDH1.

\section{Immunostaining}

Sections that were cut and mounted on positively charged slides were dried at $80^{\circ} \mathrm{C}$ for $15 \mathrm{~min}$ and then processed on a Ventana BenchMark XT immunostainer. Following $60 \mathrm{~min}$ pre-treatment with 
cell conditioner 2 ( $\mathrm{pH} 6)$, the slides were incubated with 1:30 diluted $\mathrm{H} 09$ antibody at $37^{\circ} \mathrm{C}$ for 32 min. Antibody incubation was then followed by Ventana standard signal amplification, UltraWash. Counterstaining was done with single drop of hematoxylin for $4 \mathrm{~min}$ and another drop of bluing reagent for another $4 \mathrm{~min}$. UltraView TMU universal DAB Detection Kit (Ventana) was applied for chromogenic detection. The slides were finally removed from the immunostainer and mounted.

\section{Evaluation of IDH1 immunostaining}

Acytoplasmicand perinuclear immunoreactions for IDH 1 were scored as positive.

According to Mellai et al. [13], the IDH 1 immunoreactivity is cytoplasmic and perinuclear. In portion of cases, the staining is diffuse in the glial fibrillary network. In few cases, faint nuclear staining can be detected, although IDH1 physiologically exists in the cytoplasm and peroxisomes. Endothelial cells served as good negative control.

\section{Statistical methods}

Statistical analysis was conducted using Statistical Package for the Social Sciences, Version 25 for Windows. Continuous variables were analyzed as mean values \pm standard deviation or median (range) as appropriate. Percentages were used for calculation of categorical data. Differences were analyzed with Chisquare $\left(\chi^{2}\right)$ test and Fisher's exact test when appropriate for categorical variables. Student's t-test was applied for measuring differences among continuous variables with normal distribution. Multivariate data were analyzed using logistic regression. $p<0.05$ was considered statistically significant. Kaplan-Meier estimates were used to predict the OS and PFS, as compared using log-rank tests.

\section{Results}

Our study population consisted of 60 samples. Of those, 52 patients were diagnosed as glioblastoma WHO Grade IV (86.7\%), 5 patients were diagnosed as anaplastic astrocytoma WHO Grade III (8.3\%), and 3 were diagnosed as oligodendroglioma WHO Grade III (5\%). All clinicopathologic data of the studied patients are summarized in Table 1. Correlation of IDH1 immunostaining results with other clinicopathologic parameters is shown in Table 2.

IDH1 immunohistochemical expression was positive in $46.67 \%$ of the studied high-grade glioma cases. A statistically significant relationship was detected between IDH1 expression and tumor histologic type as $100 \%$ of anaplastic oligodendrogliomas cases (Figure
Table 1: Clinicopathological characteristics of the studied cohort

\begin{tabular}{|c|c|}
\hline Clinicopathological variables & $\mathrm{n}(\%)$ \\
\hline \multicolumn{2}{|l|}{ Gender } \\
\hline Male & $32(53.3)$ \\
\hline Female & $28(46.7)$ \\
\hline \multicolumn{2}{|l|}{ Age (years) } \\
\hline Median (average) & $48(13.8)$ \\
\hline \multicolumn{2}{|l|}{ Tumor site } \\
\hline Right side hemisphere & $23(38.4)$ \\
\hline Left side hemisphere & $37(61.6)$ \\
\hline \multicolumn{2}{|l|}{ Motor symptoms } \\
\hline Present & $34(56.7)$ \\
\hline Not & $26(43.3)$ \\
\hline \multicolumn{2}{|l|}{ Sensory symptoms } \\
\hline Present & $6(10)$ \\
\hline Not & $54(90)$ \\
\hline \multicolumn{2}{|l|}{ Intracranial tension } \\
\hline Increased & $45(75)$ \\
\hline Not & $15(25)$ \\
\hline \multicolumn{2}{|l|}{ PS } \\
\hline Good & $50(83.3)$ \\
\hline Poor & $10(16.7)$ \\
\hline \multicolumn{2}{|l|}{ WHO grades } \\
\hline Grade III & $8(13.3)$ \\
\hline Grade IV & $52(86.7)$ \\
\hline \multicolumn{2}{|l|}{ Histologic type } \\
\hline Anaplastic astrocytoma & $5(8.3)$ \\
\hline Anaplastic oligodendroglioma & $3(3 \%)$ \\
\hline Glioblastoma & $52(86.7)$ \\
\hline \multicolumn{2}{|l|}{ IDH expression } \\
\hline Positive & $28(46.67)$ \\
\hline Negative & $32(53.33)$ \\
\hline \multicolumn{2}{|l|}{ Radiotherapy treatment } \\
\hline Received & $57(95)$ \\
\hline Not received & $3(5)$ \\
\hline \multicolumn{2}{|l|}{ Adjuvant chemotherapy } \\
\hline Received & $47(78.3)$ \\
\hline Not received & $13(21.7)$ \\
\hline \multicolumn{2}{|l|}{ Mortality status } \\
\hline Alive & $28(46.7)$ \\
\hline Dead & 32 (53.3) \\
\hline
\end{tabular}

Table 2: Correlation of IDH1 immunohistochemical expression with clinicopathologic variables of glioma cases

\begin{tabular}{llll}
\hline Clinicopathologic variables & $\begin{array}{l}\text { IDH1 } \\
\text { positive (\%) }\end{array}$ & $\begin{array}{l}\text { IDH1 } \\
\text { negative (\%) }\end{array}$ & p-value \\
\hline Histologic type & $4(80)$ & $1(20)$ & 0.03 \\
$\quad$ Anaplastic astrocytoma & $3(100)$ & $0(0)$ & \\
$\quad$ Anaplastic oligodendroglioma & $21(40.4)$ & $31(59.6)$ & \\
$\quad$ Glioblastoma & & & \\
Radiotherapy response & $5(38.5)$ & $8(61.5)$ & 0.019 \\
$\quad$ Progressive & $0(0)$ & $4(100)$ & \\
Stationary & $18(56.3)$ & $14(43.7)$ & \\
Partial remission & $7(87.5)$ & $1(12.5)$ & $<0.001$ \\
$\quad$ Complete remission & & & \\
Six-month response to chemotherapy & $2(11.1)$ & $16(88.9)$ & $<0.001$ \\
$\quad$ Progressive & $3(37.5)$ & $5(62.5)$ & \\
Stationary & $10(83.3)$ & $2(16.7)$ & \\
$\quad$ Partial remission & $8(88.9)$ & $1(11.1)$ & \\
$\quad$ Complete remission & & & \\
One-year response to chemotherapy & $2(10.5)$ & $17(89.5)$ & \\
$\quad$ Progressive & $6(50)$ & $6(50)$ & \\
Stationary & $3(100)$ & $0(0)$ & \\
$\quad$ Partial remission & $12(92.3)$ & $1(8.3)$ & \\
$\quad$ Complete remission & & & \\
Survival status & $25(92.6)$ & $3(7.4)$ & \\
Alive & $5(21.74)$ & $27(78.26)$ & \\
Died & & &
\end{tabular}

$3 b$ ) and $80 \%$ of the anaplastic astrocytoma cases (Figure 3a) were IDH1 positive while only $40.4 \%$ of glioblastoma cases (Figure 4 ) were IDH1 positive $(p=0.03)$. In addition, patients who expressed IDH1were in a better category of response to radiotherapy ( $p=0.019)$, and far more in a better category of 6 months and 1 year response to chemotherapy for all grades (III and IV) enrolled in this study $(p<0.001)$. Moreover, patients who expressed IDH1 had prolonged OS and PFS than those who showed negative IDH1 (Figure 5) expression for all grades (III and IV) enrolled in this study ( $<<0.001)$, Figures 1 and 2 . 


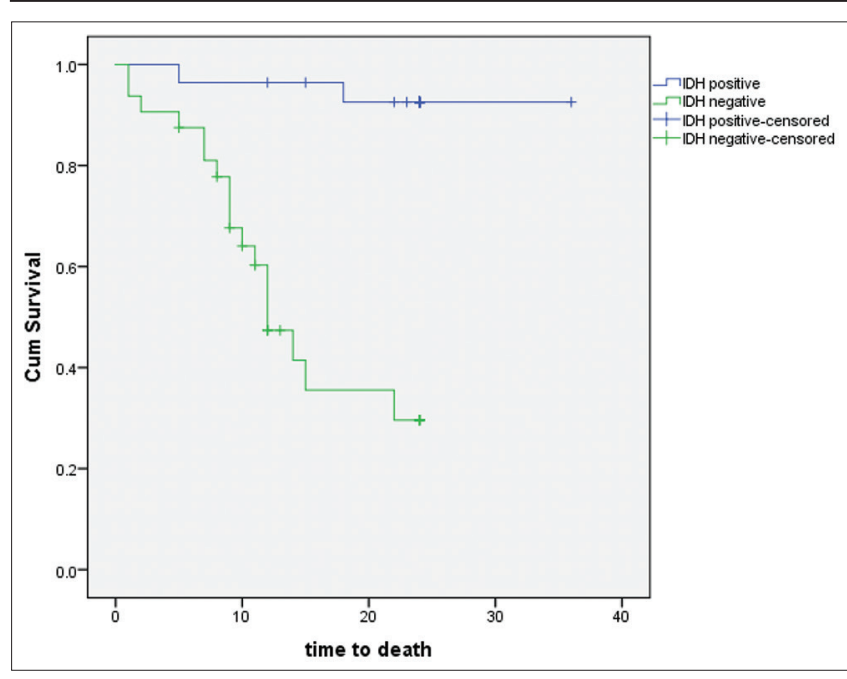

Figure 1: The relationship between isocitrate dehydrogenase 1 expression and overall survival in high-grade glioma cases using Kaplan-Meier curves

No significant correlation was detected in the current work between IDH1 immunohistochemical expression and patients age $(p=0.089)$, sex $(p=0.628)$, tumor site $(p=0.356)$, presence of motor symptoms $(p=0.134)$, sensory symptoms $(p=0.201)$, and increased intracranial tension $(p=1.0)$.

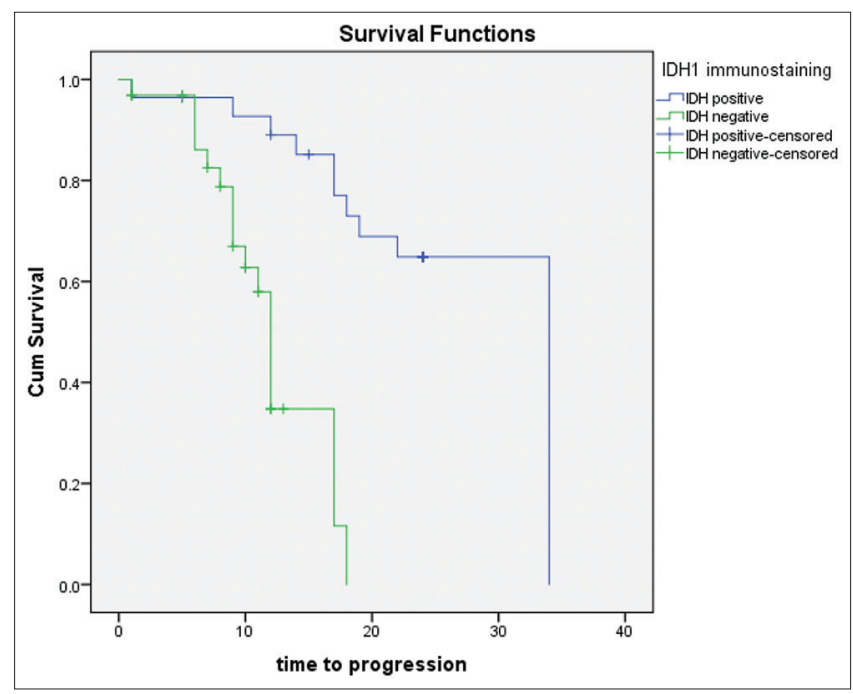

Figure 2: The relationship between isocitrate dehydrogenase 1 expression and progression-free survival (progression time) in high-grade glioma cases using Kaplan-Meier curves

\section{Discussion}

A glioma is a variant of tumor that occurs first in the brain or spine. It is termed gliomas it originates from glial cells. The majority of gliomas are located in the brain [14]. Gliomas account for nearly $30 \%$ of all brain and CNS tumors and about $80 \%$ of all primary malignant brain tumors [15].

Anaplastic astrocytoma is regarded as Grade III invasive astrocytoma, intermediate between lower grade diffuse astrocytoma (Grade II) and higher Grade IV glioblastoma [1].

Glioblastoma is an aggressive malignant astrocytic glioma that either arises de novo or on top of lower grade diffuse astrocytoma and anaplastic astrocytoma. Glioblastoma is the most fatal and aggressive primary brain tumor, with a mortality rate that nearly reaches $100 \%$ and a median survival of $<24$ months [16].

In the last formulation of CNS tumors by the WHO in 2016, Grade II diffuse astrocytomas, Grade III anaplastic astrocytomas, and Grade IV glioblastomas are reclassified into IDH mutant, IDH wild type, and NOS variants [1], [2]

In this study, a total of 60 high-grade glioma cases were enrolled; 52 cases were WHO Grade IV glioblastoma (52/60, 86.7\%), 5 anaplastic astrocytoma cases $(5 / 60,8.3 \%)$, and 3 oligodendroglioma cases $(3 / 60,5 \%)$.

In the current work, among the studied patients with high-grade gliomas, the age was a normally distributed variable with mean (47.25) and median (48) years. Patients aged $45-50$ years had the highest percentage $(21.7 \%)$. Males constituted $53.3 \%$ of the studied cases while $46.7 \%$ were females, with male-tofemale ratio 1.1:1.

IDH1 immunohistochemical expression was positive in $46.7 \%$ of the enrolled glioma cases and negative in $53.3 \%$.

Among the studied glioma cases in this study, Grade III anaplastic astrocytoma and Grade III anaplastic oligodendroglioma were highly associated with IDH1 mutation than the higher grade IV glioblastoma with statistically significant results $(p=0.03)$. This is similar to what was reported by Mellai et al. [13], that in adult patients, IDH1 mutations are significantly and inversely associated with the histological malignancy grade.

Moreover, a subset of glioblastoma cases $(40.4 \%)$ was reported to be IDH1 mutant in the present work as they positively expressed IDH1. Other studies also reported that a percentage of their patients with secondary glioblastoma were positive for IDH1 mutation [13]. However, in the present study, glioblastoma could not be classified to primary or secondary due to deficient data.

In the present study, glioma patients who expressed IDH1 are 4.15 times more likely to be in a better category of response to radiotherapy than those who showed negative IDH1 expression. The relationship between IDH1 expression and the response to radiotherapy is highly significant $(p=0.019)$. Similar findings were addressed by Li et al. [17], as in vitro induction of mutated IDH1 increased the sensitivity of gliomas to radiation.

Regarding the response to chemotherapy, patients who expressed IDH1 are 21.39 times more likely to be in a better category of response to chemotherapy than those who showed negative IDH1 
expression. The relationship between IDH1 expression and the 6-month response to chemotherapy is highly significant $(p<0.001)$.

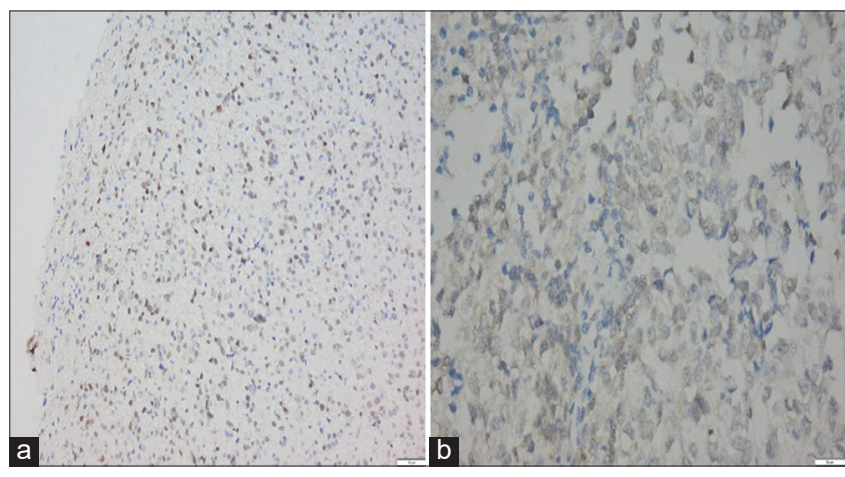

Figure 3: (a) Positive isocitrate dehydrogenase 1 (IDH1) immunohistochemistry (IHC) staining in anaplastic astrocytoma Grade III (DAB × 200). (b) Positive IDH1 IHC staining in anaplastic oligodendroglioma Grade III $(D A B \times 400)$

In addition, patients who expressed IDH1 are 30 times more likely to be in a better category of response to chemotherapy than those who showed negative IDH1 expression after 1 year response. The relationship between IDH1 expression and the 1-year response to chemotherapy is highly significant $(p<0.001)$.

These findings are also similar to what was stated by Hartmann, Combs et al., and SongTao et al. [18], [19], [20]. They stated that all diffusely infiltrating astrocytic tumors show better prognosis. Higher sensitivity to radiotherapy and concurrent temozolomide in IDH mutant glioblastoma cases was noted by Tran et al. [21]. Tateishi and Yamamoto [22] also agreed with these results. They mentioned that IDH1 status is a reliable prognostic factor. However, they stated that there could be associative molecular markers that could predict the prognosis rather than the IDH status. They stated that CDKN2A/B homozygous deletion, PIK3R1 mutation, NOTCH pathway inactivation, and PI3K pathway activation can associated with poor prognosis. These previous results are partially in agreement to what was reported by van den Bent et al. [23], that IDH1/2 mutations, $1 p / 19 q$ codeletion, and MGMT promoter methylation are pivotal prognostic factors in anaplastic oligodendroglial tumors. However, this study concluded that IDH1 mutations are not a predictive marker for the outcome to adjuvant chemotherapy.

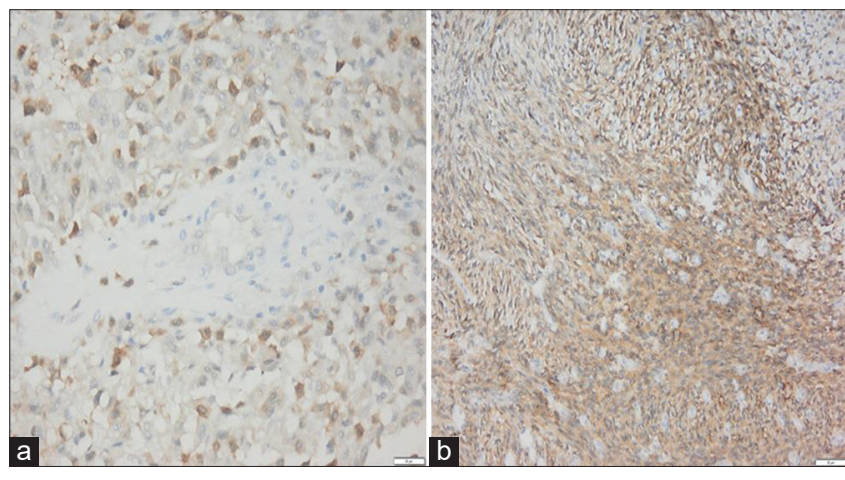

Figure 4: (a) Positive isocitrate dehydrogenase 1 (IDH1) immunohistochemistry (IHC) staining in glioblastoma Grade IV (DAB $\times 400$ ), (b) Positive IDH1 IHC staining in glioblastoma Grade IV (DAB $\times 200)$

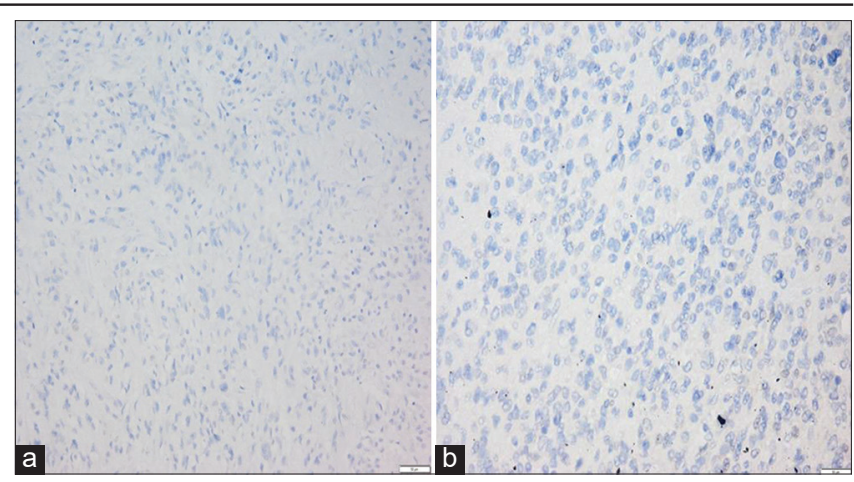

Figure 5: (a) Negative isocitrate dehydrogenase 1 (IDH1) immunohistochemistry (IHC) staining in glioblastoma Grade IV (DAB $\times$ 200), (b) Negative IDH1 IHC staining in glioblastoma Grade IV $(D A B \times 400)$

In the highlight of the current study results, patients with Grade III and IV gliomas who were IDH1 mutant are more likely to have longer OS and PFS than those who showed negative IDH1 expression. The relationship between IDH1 expression and the mortality is highly significant $(p<0.001)$. In a prospective analysis by Sanson et al. [7], Grade II-IV glioma patients whose tumors harbored mutant IDH1 had significantly longer OS than patients free of IDH1 mutation. These results have been confirmed independently by others; Combs et al. [19], Weller et al. [24], and Yan et al. [25]. Moreover, these findings are also similar to what was stated by Polívka et al. [26] who proved that the presence of IDH1 R132H mutation in the tumor tissue serves as positive prognostic factor for patients with glioblastoma in relation to PFS as well as OS. Parsons et al. [27], Tateishi and Yamamoto [22], also found that IDH1 mutant glioblastoma patients showed 3-fold longer survival than those with IDH1 wild type. Prolonged median OS of IDH mutant cases was noted by Liu et al. [28] and Yan et al. [25]. They stated that patients with IDH mutated disease showed 65-month median OS as compared to 20-month median OS of patients with IDH wild-type disease. Furthermore, Sanson et al. [7] and Liu et al. [28] stated that PFS was improved among patients with IDH mutant glioblastoma. On the other hand, no statistically significant difference in OS by the IDH1 mutation status was observed by Tabei et al. [29]. Their results showed that although IDH1 mutation has been demonstrated as a prognostic factor for diffuse gliomas including GBM that was not the case for recurrent glioblastoma that has progressed after initial treatments. OS of the patients with IDH mutant glioblastoma after the first progression from primary glioblastoma or from low-grade gliomas was similar to that of those with IDH wild type.

No significant correlation was detected in the studied glioma cases between IDH1 expression and the patients age $(p=0.089)$, the $\operatorname{sex}(p=0.628)$, the tumor site $(p=0.356)$, the presence of motor symptoms $(p=0.134)$, sensory symptom $(p=0.201)$, or increased intracranial tension $(p=1.0)$. These results are in concordance with what was reported in several studies by Jaiswal et al. [30], Shaban et al. [31], and 
Wang et al. [32]. However, these findings are different from what was stated by Hartmann [18]. They found a statistical relationship between IDH status and the patients age and the tumor site. They stated that IDH1/2 mutant cases were younger in age. Most of the tumors were frontal as opposed to temporal or parietal sites.

\section{Limitations}

The limitations of this study were not applying molecular analysis for IDH1 evaluation due to the limitations of financial resources.

\section{Conclusions}

To sum up, according to our results, IDH1 immunohistochemical expression is more detected in WHO Grade III gliomas, including anaplastic astrocytoma and anaplastic oligodendroglioma than in the WHO Grade IV glioblastoma which support its relative involvement in early gliomagenesis. Moreover, IDH1 expression is associated with better response to radiotherapy and adjuvant chemotherapy treatment. In the same context, patients who were IDH1 mutant had longer OS and longer PFS, thus suggesting IDH1 as a good prognostic marker for gliomas and a good predictive marker for response to treatment and can be a promising target for therapy in high-grade gliomas through the emerging IDH1 inhibitors.

Finally, in this work, immunohistochemical testing for IDH1 was introduced as a practical and costeffective method that can replace DNA sequencing and should be analyzed in all glioma cases.

Further study on a larger sample size is recommended to validate the current results. The application of molecular analysis to detect IDH1 mutation is also recommended for comparative purpose with the current results of $\mathrm{IHC}$ and to be able to precisely detect the IDH1 wild-type variant.

\section{References}

1. Louis DN, Ohgaki H, Wiestler OD. WHO Classification of Tumours of the Central Nervous System. $4^{\text {th }}$ ed., Vol. 1. Geneva: World Health Organization; 2016. p. 10-76.

2. Chen L, Voronovich Z, Clark K, Hands I, Mannas J, Walsh M, et al. Predicting the likelihood of an isocitrate dehydrogenase 1 or 2 mutation in diagnoses of infiltrative glioma. Neuro Oncol. 2014;16(11):1478-83. https://doi.org/10.1093/neuonc/nou097 PMid:24860178

3. Bady P, Sciuscio D, Diserens AC, Bloch J, van den Bent MJ, Marosi C, et al. MGMT methylation analysis of glioblastoma on the Infinium methylation BeadChip identifies two distinct $\mathrm{CpG}$ regions associated with gene silencing and outcome, yielding a prediction model for comparisons across datasets, tumor grades, and CIMP-status. Acta Neuropathol. 2012;124(4):54760. https://doi.org/10.1007/s00401-012-1016-2

PMid:22810491

4. Etcheverry A, Aubry M, de Tayrac M, Vauleon E, Boniface $\mathrm{R}$, Guenot $\mathrm{F}$, et al. DNA methylation in glioblastoma: Impact on gene expression and clinical outcome. BMC Genomics. 2010;11:701. https://doi.org/10.1186/1471-2164-11-701 PMid:21156036

5. Everhard S, Tost J, El Abdalaoui $\mathrm{H}$. Identification of regions correlating MGMT promoter methylation and gene expression in glioblastomas. Neuro Oncol. 2009;11(4):348-56. https://doi. org/10.1215/15228517-2009-001

PMid:19224763

6. Hartmann C, Hentschel B, Wick W. Patients with IDH1 wild type astrocytomas exhibit worse prognosis than IDH1-mutated glioblastomas, and IDH1 mutation status accounts for the unfavorable prognosis effect of higher age: Implications for classification of gliomas. Acta Neuropathol. 2010;120(6):70718. https://doi.org/10.1007/s00401-010-0781-z PMid:21088844

7. Sanson M, Marie Y, Paris S, Idbaih A, Laffaire J, Ducray F. Isocitrate dehydrogenase 1 codon 132 mutation is an important prognostic biomarker in gliomas. J Clin Oncol. 2009;27(25):41504. https://doi.org/10.1200/jco.2009.21.9832 PMid: 19636000

8. Wick W, Meisner C, Hentschel B. Prognostic or predictive value of MGMT promotor methylation in gliomas depends on IDH1 mutation. Neurology. 2013;81(17):1515-22. https://doi. org/10.1212/wnl.0b013e3182a95680

PMid:24068788

9. Loussouarn D, Le Loupp AG, Frenel JS. Comparison of immunohistochemistry, DNA sequencing and allele-specific PCR for the detection of IDH1 mutations in gliomas. Int J Oncol. 2012;40(6):2058-62. https://doi.org/10.3892/ijo.2012.1404 PMid:22447191

10. Perrech M, Dreher L, Röhn G. Qualitative and quantitative analysis of IDH1 mutation in progressive gliomas by allelespecific qPCR and Western blot analysis. Technol Cancer Res Treat. 2019;18:1533033819828396. https://doi. org/10.1177/1533033819828396 PMid:30943868

11. West $\mathrm{H}$, Jin J. JAMA Oncology Patient Page. Performance status in patients with cancer. JAMA Oncol. 2015;1(7):998. PMid:26335750

12. Kelly C, Majewska P, loannidis S, Raza MH, Williams M Estimating progression-free survival in patients with glioblastoma using routinely collected data. J Neurooncol. 2017;135(3):6217. https://doi.org/10.1007/s11060-017-2619-1 PMid:28956223

13. Mellai M, Caldera V, Annovazzi L, Schiffer D. The distribution and significance of IDH mutations in gliomas. Genes Chromosomes Cancer. 2013;34:416-2. https://doi.org/10.5772/52357

14. Mamelak AN, Jacoby DB. Targeted delivery of antitumoral therapy to glioma and other malignancies with synthetic chlorotoxin (TM-601). Expert Opin Drug Deliv. 2007;4(2):17586. https://doi.org/10.1517/17425247.4.2.175 PMid: 17335414

15. Goodenberger $M L$ and Jenkins RB. Genetics of adult glioma. Cancer Genet. 2012;205(12):613-21. https://doi.org/10.1016/j. cancergen.2012.10.009

PMid:23238284 
16. DeAngelis LM. Brain tumors. N Engl J Med. 2001;344(2):114-23. PMid:11150363

17. Li S, Chou AP, Chen W. Overexpression of isocitrate dehydrogenase mutant proteins renders glioma cells more sensitive to radiation. Neuro Oncol. 2013;15(1):57-68. https:// doi.org/10.1093/neuonc/nos261

PMid:23115158

18. Hartmann, C. Long-term survival in primary glioblastoma with versus without isocitrate dehydrogenase mutations. Clin Cancer Res. 2013;19(18):5146-57. https://doi.org/10.1158/1078-0432. ccr-13-0017 PMid:23918605

19. Combs SE, Rieken S, Wick W. Prognostic significance of IDH-1 and MGMT in patients with glioblastoma: One step forward, and one step back? Radiat Oncol. 2011;6:115. https://doi. org/10.1186/1748-717x-6-115 PMid:21910919

20. SongTao Q, Lei $Y$, Si G, YanQing D, HuiXia H, XueLin Z. IDH mutations predict longer survival and response to temozolomide in secondary glioblastoma. Cancer Sci 2012;103(2):269-73. https://doi.org/10.1111/j.1349-7006.2011.02134.x PMid:22034964

21. Tran AN, Lai A, Li S, Pope WB, Teixeira S, Harris RJ. Increased sensitivity to radiochemotherapy in IDH1 mutant glioblastoma as demonstrated by serial quantitative MR volumetry. Neuro Oncol. 2014;16(3):414-20. https://doi.org/10.1093/neuonc/ not198 PMid:24305712

22. Tateishi K, Yamamoto T. IDH-Mutant Gliomas. London: IntechOpen; 2019. p. 84543.

23. Van den Bent MJ, Dubbink HJ, Marie Y. IDH1 and IDH2 mutations are prognostic but not predictive for outcome in anaplastic oligodendroglial tumours: A report of the European organization for research and treatment of cancer brain tumour group. Clin Cancer Res. 2010;16(5):1597-604. https://doi. org/10.1158/1078-0432.ccr-09-2902 PMid:20160062

24. Weller M, Felsberg J, Hartmann C. Molecular predictors of progression-free and overall survival in patients with newly diagnosed glioblastoma: A prospective translational study of the German Glioma Network. J Clin Oncol. 2009;27(34):5743-50. https://doi.org/10.1200/jco.2009.23.0805

PMid:19805672

25. Yan, H, Parsons D.W, Jin G. IDH1 and IDH2 Mutations in gliomas. N Engl J Med 2009;360(8):765-73.

PMid:19228619

26. Polívka J, Pešta M, Pitule P, Hes O, Holubec L, Polívka J, et al. IDH1 mutation is associated with lower expression of VEGF but not microvessel formation in glioblastoma multiforme. Oncotarget. 2018;9(23):16462-76. https://doi.org/10.18632/ oncotarget.24536

PMid:29662659

27. Parsons DW, Jones S, Zhang X. An integrated genomic analysis of human glioblastoma multiforme. Science. 2008;321(5897):1807-12.

PMid:18772396

28. Liu Y, Lang F, Chou FJ, Zaghloul KA, Yang C. Isocitrate dehydrogenase mutations in glioma: Genetics, biochemistry, and clinical indications. Biomedicines. 2020;8(9):294. https:// doi.org/10.3390/biomedicines8090294 PMid:32825279

29. Tabei $Y$, Kobayashi K, Saito K. Survival in patients with glioblastoma at a first progression does not correlate with isocitrate dehydrogenase (IDH) 1 gene mutation status. Jpn $J$ Clin Oncol 2021;51:45-53. https://doi.org/10.1093/jjco/hyaa162

30. Jaiswal S, Chaudhary N, Prasad P. Expression of isocitrate dehydrogenase-1 (IDH-1) mutant protein in gliomas. Tech Neurosurg Neurol. 2018;1(3):1-6.

31. Shaban ZM, Al-Aubaidy SR, Hameedi AD. IDH1 mutation in gliomas in Baghdad by immunohistochemical study. Int J Genet Genomics 2018;6(1):1-7.

32. Wang PF, Liu N, Song HW. IDH-1R132H mutation status in diffuse glioma patients: Implications for classification. Oncotarget. 2016;7(21):31393-400. https://doi.org/10.18632/ oncotarget. 8918

PMid:27120786 